Branch”(C2020.

Available online

\title{
RISK FACTORS FOR PERIODONTAL DISEASE- A REVIEW
}

\author{
Dr. Arunagiri, Dr. Anil Melath, Dr. Mohammed Feroz, Dr. Subair \\ Department of Periodontology, \\ Mahe Institute of Dental Sciences and Hospital, Chalakkara, Palloor, Mahe, Pondicherry, \\ India
}

\section{To access $\&$ cite this article}

Website: jidam.idamadras.com

DOI:10.37841/jidam_2020_V7_I4_03

\section{Address for Correspondence:}

Dr. Arunagiri,

Postgraduate Student, Department of Periodontology,

Mahe Institute of Dental Sciences and Hospital,

Chalakkara, Palloor, Mahe - 673310

Email id: giriarun133@gmail.com

$\begin{array}{ll}\text { Received } & : 06.09 .2020 \\ \text { Accepted } & : 15.09 .2020 \\ \text { Published } & : 27.09 .2020\end{array}$

\section{ABSTRACT}

A probability or threat of damage, injury, liability, loss, or any other negative occurrence that is caused by external or internal vulnerabilities, and that may be avoided through pre- emptive action is called as risk. This article explains about the evidence on the potential role of modifiable and non-modifiable risk factors associated with periodontal disease. It is important to understand the etiological factors and the pathogenesis of periodontal disease to recognize and appreciate the associated risk factors. As periodontal disease is multifactorial, effective disease management requires a clear understanding of all the associated risk factors. The local factors include pre-existing disease as evidenced by deep probing depths and plaque retention areas associated with defective restorations. Systemic risk factors recently have been identified by large epidemiologic studies using multifactorial statistical analyses to correct for confounding or associated co-risk factors. The study of risk in periodontal disease is a rapidly emerging field and much is yet to be learned.

KEYWORDS: Periodontal disease, risk factors, host response, risk assessment 
Periodontal disease is a group of persistent infections induced via pathogenic microorganisms that colonize the periodontium. The onset and progression of periodontal infections are influenced through local and systemic conditions. Local elements consist of dental plaque and retention areas of the plaque, such as dental calculus and faulty restorations. Systemic risk elements consist of poorly managed diabetes mellitus and smoking. Systemic prerequisites related with immunodeficiency, such as neutropenia, AIDS

/ HIV infections, are additionally essential hazard factors. Recent research has published countless probably necessary periodontal risk indicators ${ }^{1}$. These consist of stress and coping behaviors and osteopenia related with estrogen deficiency. There are additionally demographic elements related with periodontal disease, which include gender, and hereditary factors.

\section{TERMINOLOGIES:}

Risk (Kleinbaum 1982): It is the probability that an individual develops a certain disease or exposure or changes the state of health in a given period.

Risk factor: - It is an environmental exposure, a behavioral aspect or an intrinsic characteristic associated with a disease. - To be identified as a risk factor, exposure must occur before the onset of the disease ${ }^{17}$.

Risk indicators: - They are probable and putative risk factors that have been identified in cross-sectional studies but not confirmed through longitudinal studies. - They are not believed to be part of the causal chain.

Markers or risk predictors: These are characteristics that have the ability to predict high risk individuals, but are not part of the causal chain. They can predict the future course of the disease. Risk determinants: These forms, sometimes replaced by the term risk factor, should be reserved for those risk factors that cannot be changed, are probably not in the causal chain and are not the objectives of the intervention. Risk assessment: - It is the process of determining the quantitative and qualitative probability of adverse events that can result from exposure to specific health risks or the absence of beneficial influences.

\section{RISK FACTORS OF PERIODONTAL DISEASE}

MODIFIABLE RISK FACTORS:

\section{MICROORGANISMS AND PERIODONTAL DISEASE;}

Of all a variety of microorganisms that colonize the mouth, there are three, Porphyromonas gingivalis, Tannerella forsythia (formerly Bacteroides forsythus) and Actinobacillus actinomycetemcomitans which have been implicated as causative agents in periodontitis ${ }^{2}$.

\section{SMOKING:}

Cross-sectional and longitudinal records provide strong aid that the risk of growing periodontal disease measured by means of loss of clinical attachment and loss of alveolar bone will increase with increasing smoke ${ }^{3}$. Studies have proven that smoking no longer minimizes the amount of plaque existing and, in fact, smokers may additionally experience much less gingival bleeding than nonsmokers with decrease in plaque indices. It has been suggested that this reflects an alteration in the size of the blood vessels which makes the gingival tissues perfect. It has also been suggested that the reduction in bleeding reflects an underlying disruption of the immune response and that this could explain the greater loss of clinical attachment and alveolar bone ${ }^{4}$.

\section{DIABETES MELLITUS:}

One of the important oral signs of diabetes is gingivitis and periodontitis. Both type 1 and type 2 diabetes mellitus are related with excessive degrees of systemic markers of inflammation. The excessive inflammatory status in diabetes contributes to microvascular and macrovascular issues and it is clear that hyperglycemia can trigger the activation of pathways that enlarge inflammation, oxidative stress and apoptosis ${ }^{5}$. Elevated serum IL-6 and TNF- $\alpha$ ranges have been validated in diabetes and obesity, and serum IL-6 and C-reactive protein (CRP) ranges have been proven to predict the future onset of diabetes mellitus type 2 . High ranges of CRP are additionally related with insulin resistance, type two diabetes mellitus and cardiovascular disease. TNF$\alpha$ and IL-6 are the 
primary inducers of acute phase proteins, including CRP and each have been shown to alter the signaling of intracellular insulin, which may additionally contribute to insulin resistance. Serum IL-6 and CRP levels are additionally increased in patients with periodontitis, with IL-6 levels associated to the spread of the disease. Systemic irritation associated with periodontal disease can consequently enhance the diabetic status. Adipokines may additionally also contribute to susceptibility to both periodontitis and diabetes, and the proinflammatory properties of leptin may additionally be especially essential in regulating periodontal inflammation in overweight and / or type two diabetes mellitus ${ }^{6}$.

\section{CARDIOVASCULAR DISEASE:}

The biological plausibility of the association between periodontal diseases and cardiovascular diseases is well studied and it includes some of the following possible mechanisms: high concentrations of cholesterol and the action of oral bacteria in the process of atherosclerosis or the participation of acute-phase proteins that may increase in chronic periodontitis ${ }^{7}$.Varied biological mechanisms have been proposed to provide an explanation for the relationship between periodontal and cardiovascular diseases. Therefore, periodontitis can probably elicit a systemic inflammatory response and it deserves more attention. Periodontal disease is capable of predisposing to vascular diseases due to the prosperous supply of subgingival microbial species and the host's response. Furthermore, we must be aware that these diseases share many risk factors and there are evident similarities to the basic pathogenic mechanisms ${ }^{8}$. Periodontitis is associated with increase in the level of C-reactive protein and fibrinogen, irrespective of coronary diseases. Furthermore, there is evidence that suggests that the increase in the levels of systemic markers of inflammation, such as the C-reactive protein (CRP) and interleukin-6 (IL- 6), is associated with cardiovascular diseases.

Bacteremia from periodontitis and dental disease is known to be the primary cause of infective endocarditis. In particular, patients who have undergone heart valve surgery have a significant risk of life-threatening infective endocarditis. Epidemiological and microbiological studies have lent credence to the concept that periodontal disease may be a separate risk factor for cardiovascular disease, cerebrovascular disease, and preterm delivery of low birth weight infants.

Wu et al ${ }^{9}$ have proven that periodontal disease is any other putative and independent risk element for cerebrovascular disease, specifically for ischemic stroke. In 1989, Kimmo Mattila and his co-workers in Finland conducted two separate case control studies totaling 100 patients with acute myocardial infarction and they compared these patients with 102 control subjects selected from the community. A dental examination was performed on all the patients and a dental index was computed. In this original report, subjects with evidence of oral infection were 30\% more likely to present with myocardial infarction as against subjects without oral infections. Janket et al performed a meta-analysis of nine cohort studies of PD as a risk factor for future cardiovascular and cerebrovascular events RR 1.19; (95\% CI [1.08$1.32]$ ) and found an overall $19 \%$ increased risk of such events in individuals with periodontitis. The increase in risk was greater (44\%) in people under age 65. However, Garcia RI et al 2010 and Trevisan M, Dorn J et al 2010 have not discovered a relationship between periodontitis and ischemic coronary heart disease.

\section{DRUG INDUCED DISORDERS:}

Irrespective of the causative medication, the clinical appearance of drug-induced gingival overgrowth remains the same, with the onset usually occurring 1-3 months following commencement of the medication. The dosage of medication, combined with the amount of dental plaque and periodontal inflammation, is linked to the prevalence and severity of the overgrowth seen. The anterior gingiva is one of the most commonly affected sites. The overgrowth begins normally in the interdental papilla region and spreads to cover the buccal and palatal surfaces of the teeth. Although largely a cosmetic concern, due to the overgrowth causing a reduction in clinical crown height and, in some cases, completely obscuring the teeth; the enlargement can also cause speech and masticatory difficulties, especially in young children. Fibrosis can occur in chronic overgrowth cases, leading to tooth migration and possible secondary malocclusion in the presence of altered masticatory habits. Enlargement of the interdental papillae can also lead to displacement of teeth and thus resulting in diastema. Although the gingival enlargement is 
not directly harmful, inflammatory changes become apparent due to the difficulties in plaque control, leading to oedema, erythema and bleeding. This appearance is commonly encountered in the presence of preexisting periodontal disease.

Some drugs drastically minimize salivary flow. These consist of antihypertensives, narcotic pain relievers, some tranquilizers and sedatives, antihistamines and antimetabolites. Other drugs, specifically those in liquid or chewable form that contain added sugar, alter the $\mathrm{pH}$ and composition of the plaque, and facilitating adherence to the surfaces of the teeth ${ }^{10}$. Drugs can be a contributing factor to periodontal disease. Drugs such as anticonvulsants, calcium channel blocking agents and cyclosporine can result in overgrowth of the gums $^{11}$. In 1996, Seymour et al postulated the concept of genetic predisposition for DIGO etiopathology. This is proven by way of the truth that some humans develop gingival hyperplasia and others do not while taking the same drug. The ordinary inflammatory response of the gingival fibroblasts and the consequent proliferation of the connective tissue matrix underlines the heterogenetic character of the gingival fibroblasts in response to the inducer drugs. The frequent mechanism of action at the cell stage of these three extraordinary classes of tablets appears to be the inhibition of the entry of cations, in precise sodium and calcium ions. Clinicians accept the truth that gingival overgrowth is multifactorial, bacterial plaque appears to be a contributing factor and the severity of gingival overgrowth is believed to be at once proportional to the degree of plaque accumulation and plaqueinduced inflammation. The decreased transport of energetic cation-dependent folic acid (FA) inside the gingival fibroblasts reasons a decreased absorption of FAby using cells, inflicting adjustments in the matrix metalloproteinase metabolism and lack of ability to set off collagenase. This causes the accumulation of connective tissue and collagen due to the lack of collagenase triggered through DIGO.

Patients with gingival overgrowth may be at an increased risk of periodontal disease, as well as tooth decay. Gingival overgrowth creates pseudo pocketing coronal to the cementoenamel junction, which may hinder effective plaque control. In periodontally susceptible patients, this may progress to loss of periodontal attachment. Another recent study reported that patients who take nonsteroidal antiinflammatory drugs regularly are prone to the development of aphthous ulcers or lichenoid type lesions. Not only are these patients at increased risk for occurrence of these lesions, but they are more prone to recurrence of these lesions.

\section{STRESS:}

The term stress serves as a convenient description for complicated and incomplete psychological andphysiological phenomena. Anxiety, as well as different emotional or psychosocial tensions, produce properly characterized neuroendocrine and biochemical modifications in experimental animals. Patients with insufficient stress behavioral techniques (defensive coping) are at elevated chance of extreme periodontal disease ${ }^{12}$.

\section{OBESITY:}

Obesity has been stated to be a vital chance element for periodontal disease. Various explanations have been provided for the association between weight problems and periodontal disease in younger adults. Young humans can also have unique eating regimen plans than older study participants. Research on dietary developments in teens aged 11 to 18 exhibits a huge reduction in uncooked fruits and greens except potatoes, which are sources of vitamin C. In addition, teens have diminished their calcium consumption and improved consumption tender drinks instead than citrus juices. This is essential for oral fitness due to the fact that low dietary calcium and vitamin $\mathrm{C}$ consumption has been related with periodontal disease. People who eat much less than the encouraged each day allowance (RDA) for calcium and vitamin $\mathrm{C}$ have barely greater charges of periodontal disease ${ }^{13}$.

\section{NON-MODIFIABLE RISK FACTORS}

\section{OSTEOPOROSIS:}

Many of the studies conducted to date suggest there is a relationship between skeletal osteoporosis and bone loss $^{14}$ to the extent that postmenopausal osteoporosis may result in dental osteopenia involving the jaws, and particularly the mandible. Osteoporosis was once extensively related with 
extreme alveolar crestal bone loss and the occurrence of periodontitis cases in postmenopausal women. A review of the relationship between osteopenia, oral bone loss, and periodontal disease concluded that osteopenia does play a role in the expression of periodontal disease. The evaluation indicated a direct association between skeletal and mandibular osteopenia and loss of alveolar crestal height and enamel loss in postmenopausal females. Taguchi et al have stressed that it is important to distinguish among osteopenia, which has been defined in general terms as a decrease in normal mineralized bone, postmenopausal osteoporosis, which is a disease caused by the cessation of estrogen production and characterized by spinal fractures that occur between the ages of 50 and 70 years, and osteoporosis, which affects an older population and results in proximal femur fractures. Periodontitis and osteopenia may have common etiological agents that may either directly influence or modulate both disease processes $^{15}$.

\section{HEMATOLOGICAL DISORDERS:}

Hemorrhagic gingival overgrowth with or without necrosis is a frequent early manifestation of acute leukemia. Chronic leukemia patients may additionally experience similar but much less extreme periodontal changes. Chemotherapy or remedy related with bone marrow transplantation can additionally adversely have an effect on gingival health ${ }^{16}$.

\section{HOST RESPONSE:}

Chronic periodontitis includes complex interactions between microbial elements and sensitive host. Bacterial elements such as lipopolysaccharides and cytokines activate macrophages to produce cytokines such as interleukin (IL) -1 and tumor necrosis issue (TNF). These cytokines activate the fibroblasts that stay in the periodontal tissues to the matrix metalloproteinases (MMP), a plasminogen activator, which can activate the plasmin. Plasmin, in turn, can activate some other sorts of latent MMPs, while tissue metalloproteinase (TIMP) inhibitorscan inactivate active MMPs. Prolonged and excessive bacterial promotions of MMPs amongst sensitive men and women result in an enchancement in the degradation of collagen, which is a necessary factor of the periodontal matrix. MMP-8 and -9 are released from polymorphonuclear leukocytes (PMN) and are accountable for a tremendous section of the destruction precipitated through the host response. MMP-13 additionally enables bone resorption by using degrading the collagen matrix of the bone after the bone is demineralized by means of osteoclasts ${ }^{17}$.

\section{PREGNANCY:}

Offenbacher et al discovered extensively higher periodontal attachment loss amongst mothers of PLBW toddlers than mothers of term infants. Likewise, quite a few different researches have advised a poor effect of periodontal disease on the direction of pregnancy. It has been recommended that periodontal disease might also make bigger the risk of having low birth weight premature infants (PLBW). This end result is believed to be the effect of biological mediators of inflammatory methods such as prostaglandins $E_{2}$ and TNF. The frequent bacterial product lipopolysaccharide may also have a triggering function at some stage of pregnancy ${ }^{18}$.

\section{RISK ASSESSMENT:}

When risk assessment is performed without the use of a risk assessment model, there is a large degree of variation between general dentists and Periodontists and between the Periodontists themselves, so it is important to develop risk assessment models.

\section{DEVELOPMENT OF THE MODEL:}

Lang and Tonetti, $2003{ }^{19}$ model is a continuous multilevel risk assessment model that incorporates subjective assessments of dental and site risk and generates a functional diagram and, according to the area of the polygon, classifies the patient into low, medium and high-risk categories (Fig. 1).

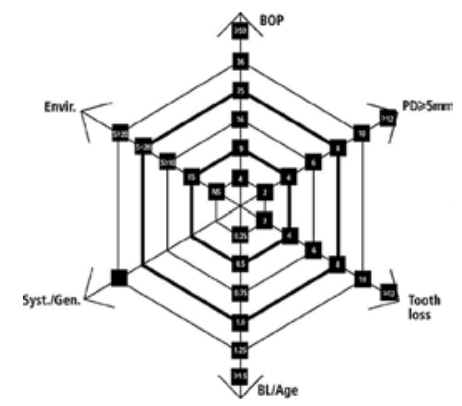

Fig 1: Lang and Tonetti's periodontal risk assessment (PRA) model (Lang and Tonetti, 2003; Lang et al, 2003). 
However, the original model has the following limitations:

- Mainly assesses the cumulative status of a patient with periodontitis

- There is no adequate identification of risk factors and risk determinants

- In the functional diagram, the presence of a systemic disease is assessed as a high-risk factor without emphasis on the current state of a disease

- Smoking is assessed in the risk assessment model, but another potential risk factor, diabetes, is not assessed separately and is included in the category of systemic diseases

- Does not take into account various dental factors, which can modify or initiate the progression of periodontal disease.

Four entities from the original risk assessment model were maintained in the new model: bleeding on probing (BOP), depth of survey, tooth loss and smoking (the latter was described under "environmental factors" in the original model). The entities that were added to the new model included various aspects of risk assessment, in particular risk factors (diabetes and dental deposits or factors that can hold deposits) and other factors that determine risk such as socioeconomic factors and stress (Page and Beck, 1997)

The risk assessment model included eight parameters:

1. Percentage of sites with BOP

2. Number of sites with probing depth (PD) $\geq 5 \mathrm{~mm}$

3. Number of missing teeth

4. Loss / attachment ratio (AL) / age

5. Diabetic state

6. Smoking

7. Dental state: interaction of systemic factors

8. Other background features

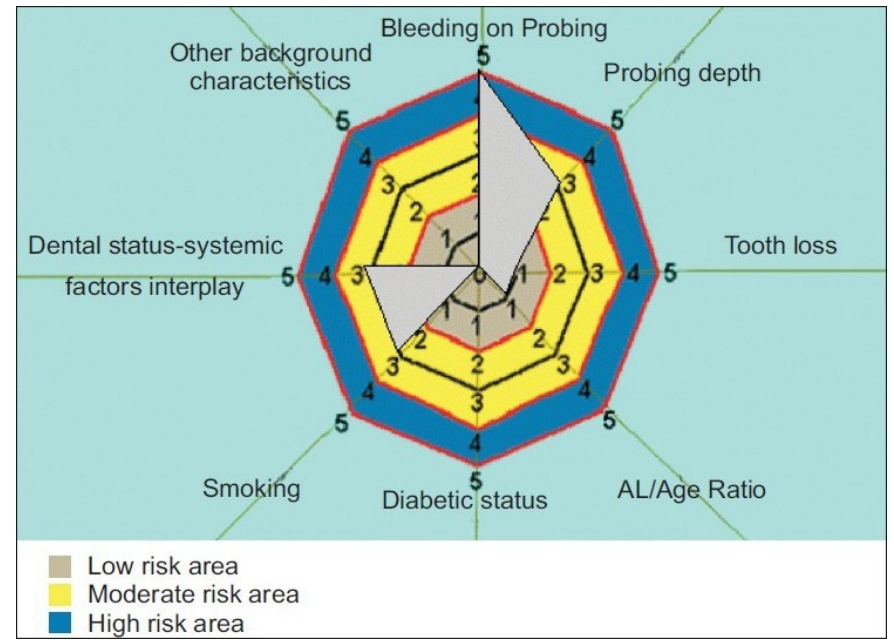

$\mathrm{BOP}, \mathrm{PD}$, tooth loss and $\mathrm{AL} /$ age ratio measure the cumulative periodontal status, which is the current state of the individual due to periodontal disease. Diabetic status and smoking are risk factors and stress and socioeconomic factors are the determinants of risk, which have been assessed in this new model. However, all of these parameters were assessed on a five-point risk scale to balance the sensitivity of the risk assessment with the time and experience required to collect the required information (Page et al, 2003) ${ }^{20}$.

\section{CONCLUSION:}

It is imperative that the dentist look beyond the oral cavity in search of factors to recommend changes in order to help his patients achieve their common goal of prevention or management of periodontal disease, and therefore possibly also improve overall health and it is also important to understand the etiological factors and the pathogenesis of periodontal disease to recognize and appreciate the associated risk factors. Since periodontal disease is multifactorial, effective disease management requires a clear understanding of all associated risk factors.

\section{FINANCIAL SUPPORT AND SPONSORSHIP:}

Nil

\section{CONFLICTS OF INTEREST:}

There are no conflicts of interest. 


\section{REFERENCES:}

1. Löe H, Anerud A, Boysen H, Smith M. The natural history of periodontal disease in man. The rate of periodontal destruction before 40 years of age. J Periodontol 1978; 49:607-20.

2. Ezzo PJ, Cutler CW. Microorganisms as risk indicators for periodontal disease. Periodontol 2000 2003; 32:24-35.

3. Van Dyke TE, Sheilesh D. Risk factors for periodontitis. J Int Acad Periodontol 2005; 7:3-7.

4. Mirbod SM, Ahing SI, Pruthi VK. Immunohistochemical study of vestibular gingival blood vessel density and internal circumference in smokers and non-smokers. J Periodontol 2001; 72:1318-23.

5. S. G. Grossi, F. B. Skrepcinski, T. DeCaro et al., "Treatment of periodontal disease in diabetics reduces glycated hemoglobin," Journal of Periodontology, vol. 68, no. 8, pp. 713-719, 1997.

6. C. J. Gustke, "Treatment of periodontitis in the diabetic patient: a critical review," Journal of Clinical Periodontology, vol. 26, no. 3, pp. 133-137, 1999.

7. D. F.Kinane and G. D. O. Lowe, "How periodontal disease may contribute to cardiovascular disease,” Periodontology 2000, vol.23, no. 1, pp. 121-126, 2000.

8. Y. Nakamura, O. Tagusari, Y. Seike et al., "Prevalence of periodontitis and optimal timing of dental treatment in patients undergoing heart valve surgery," Interactive Cardiovascular and Thoracic Surgery, vol. 12, no. 5, pp. 696-700, 2011.

9. Wu, M. Trevisan, R. J. Genco, J. P. Dorn, K. L. Falkner, and C. T. Sempos, "Periodontal disease and risk of cerebrovascular disease: The First National Health and Nutrition Examination Survey and its follow-up study," Archives of Internal Medicine, vol. 160, no. 18, pp. 2749-2755, 2000.

10. D. W. Cohen, "Periodontal medicine in the next millennium," The International Journal of Periodontics \& Restorative Dentistry, vol. 20, no. 1, pp. 6-7, 2000.
11. T.D.Rees andR. A. Levine, "Systematic drugs as a risk factor for periodontal disease initiation and progression," Compendium, vol. 16, no. 1, p. 20, 22, 26, 42, 1995.

12. Linden GJ, Mullally BH, Freeman R. Stress and the progression of periodontal disease. $\mathrm{J}$ Clin Periodontol 1996; 23:675-80.

13. Tomar SL, Asma S. Smoking-attributable periodontitis in the United States: Findings from NHANES III. National Health and Nutrition Examination Survey. J Periodontol 2000; 71:743-51.

14. R. F. Neiva, J. Steigenga, K. F. Al-Shammari, and $\mathrm{H}$. Wang, "Effects of specific nutrients on periodontal disease onset, progression and treatment,” Journal of Clinical Periodontology, vol. 30, no. 7, pp. 579-589, 2003.

15. S. Suresh, T. S. Kumar, P. K. Saraswathy, andK. H. Pani Shankar, "Periodontitis and bone mineral density among pre- and postmenopausal women: a comparative study," Journal of Indian Society of Periodontology, vol. 14, no. 1, pp. 30-34, 2010.

16. T.Waltimo, S. Christen, J. H.Meurman, and A. Filippi, "Dental care of patients with leukemia," Schweiz Monatsschr Zahnmed, vol. 115, no. 4, pp. 308-315, 2005.

17. R. J. Genco, "Current view of risk factors for periodontal diseases," Journal of Periodontology, vol. 67, no. 10, pp. 10411049, 1996.

18. Offenbacher, V. Katz, G. Fertik et al., "Periodontal infection as a possible risk factor for pretermlow birth weight," Journal of Periodontology, vol. 67, supplement 10, pp. 1103-1113, 1996.

19. Lang NP, Tonetti MS. Periodontal risk assessment (PRA) for patients in supportive periodontal therapy (SPT). Oral Health Prev Dent 2003; 1:7-16.

20. Page RC, Beck JD. Risk assessment in periodontal disease. Int Dent J 1997; 47:6187. 